\title{
Improved Time and Frequency Synchronization Algorithm for 802.11a Wireless Standard based on the SIGNAL Field
}

\author{
Cong Luong Nguyen ${ }^{1}$, Anissa Mokraoui ${ }^{1}$, Pierre Duhamel ${ }^{2}$, Nguyen Linh-Trung ${ }^{3}$ \\ ${ }^{1}$ L2TI, Institut Galilée, Université Paris 13 Sorbonne Paris Cité, 99 avenue Jean-Baptiste Clément, 93430 \\ Villetaneuse, France \\ 2 LSS/CNRS, SUPELEC, 3 rue Joliot-Curie, 91192 Gif sur Yvette, France \\ 3 Electronics and Telecommunications, University of Engineering and Technology, Vietnam National \\ University Hanoi, E3, 144 Xuan Thuy, Cau Giay, Hanoi, Vietnam
}

Correspondence: Nguyen Linh-Trung, linhtrung@vnu.edu.vn

Manuscript communication: received 10 March 2013, accepted 26 July 2013

\begin{abstract}
Time and frequency synchronization in the IEEE 802.11a OFDM (Orthogonal Frequency Division Multiplexing) wireless communication system is addressed in this paper. Usually synchronization algorithms rely only on training sequences specified by the standard. To enhance the synchronization between stations, we propose to extract known information by both the transmitter and the receiver at the IEEE 802.11a physical layer to be then exploited by the receiver in addition to the training sequences. Indeed the parts of the identified SIGNAL field are either known or predictable from the RtS (Request to Send) control frame when the CSMA/CA (Carrier Sense Multiple Access with Collision Avoidance) mechanism is triggered jointly to bit-rate adaptation algorithms to the channel. Moreover the received RtS control frame allows the receiver to estimate the channel before time synchronization stage improving then the performance of the proposed synchronization algorithm. Simulation results show that the performance of the proposed synchronization algorithm is improved as compared to existing algorithms.
\end{abstract}

Keywords- IEEE 802.11a, OFDM, SIGNAL field, channel estimation, time synchronization, frequency synchronization, RtS control frame, CSMA/CA.

This work was supported under Project 39/2012/HD/NDT granted by the Ministry of Science and Technology of Vietnam.

\section{INTRODUCTION}

In wireless communication systems, spectral efficiency always plays an important role and Orthogonal Frequency Division Multiplexing (OFDM) is one of the modulation techniques that helps increase the efficiency because it allows to transmit a large number of closely spaced orthogonal sub-carrier signals. By exploiting the OFDM technique, the IEEE 802.11a standard supports a high-speed data transmission at rates up to $54 \mathrm{Mbps}$ [1]. However, the OFDM system performance is greatly influenced by Inter-Symbol Interference (ISI) and Inter-Carrier Interference (ICI) caused by time and frequency offsets [2]. Therefore, accurate time and frequency synchronization is required before the data packet is demodulated at the receiver. A synchronization process can be performed by either exploiting some redundant information (i.e., Non-Data-Aided (NDA) techniques) or using a training sequence (i.e., DataAided (DA) techniques) included in the transmission physical packet.

NDA algorithms using the Cyclic Prefix (CP) can be found in [3], [4] and [5]. Recall that the CP is a copy of the data part of the OFDM symbol. Before being transmitted via the channel, each OFDM symbol is preceded by the $\mathrm{CP}$ to reduce the ISI effect in multipath channel environment. In [3], a Maximum Likelihood (ML) based algorithm for time and frequency synchronization is presented. The symbol timing and the Carrier Frequency Offset (CFO) parameters are defined by searching the index that maximizes the ML function. To be simpler in computation, the received complex samples can be quantized into some certain symbols. In [5], instead of working directly with the received samples, all complex samples are presented by one of four new complex samples. The new complex samples contain real and imaginary parts of values \pm 1 . In spite of such a quantization, the new complex samples still contain information about the symbol timing. In general, the CP-based algorithms have low accuracy because there usually is an ISI area included in the $\mathrm{CP}$ interval. However, they achieve a high spectral efficiency since the transmission of training symbols is not required, in contrast to the DA algorithms.

We first mention the method in [6], proposing a training sequence which is composed of two symbols for time and frequency synchronization. The first symbol consists of two identical halves. Based on these halves, the receiver realizes the Auto-Correlation Function (ACF) applied on the received signal, the maxi- 
mum absolute value of which allows us to estimate the symbol timing. Once the time synchronization is obtained, the CFO estimation is performed in two main steps. The first step estimates the Fractional Frequency Offset (FFO) using the phase of the ACF between two halves of the first symbol. The second step estimates the Integer Frequency Offset (IFO) by exploiting the second symbol. A Cross-Correlation Function (CCF) between the known second symbol and the corresponding received symbol in frequency domain is defined. The IFO value is then determined by the index that maximizes this function. To reduce the training symbols and thus get a high spectral efficiency, only one training symbol was proposed in [7] and it is generated by repeating (copying) the first symbol of the sequence of data symbols. The symbol timing and the CFO estimate are achieved via the ACF between the two repetition symbols. In [8], a joint CFO and channel estimation was proposed, using the Maximum-A-Posteriori (MAP) criterion. Specifically, a posteriori probability function of the CFO and channel coefficients is investigated. The unknown parameters are then estimated by finding the values maximizing this function.

In the IEEE 802.11a standard, training sequences have been specified at the beginning part of the physical packet (i.e., the preamble). These sequences are composed of a Short Training Field (STF) and a Long Training Field (LTF). Based on this structure, an algorithm for both time and frequency synchronization was developed in [9] where Coarse Time Synchronization (CTS) and Coarse Frequency Synchronization (CFS) use the ACF applied on the STF, while Fine Frequency Synchronization (FFS) and Fine Time Synchronization (FTS) use the ACF calculated on the LTF. The same approach is found in [10] where the FTS is carried out by the CCF between the received signal and a part of the LTF. Apart from the use of the ACF and CCF, the FTS is also combined with the channel estimation via the relationship between the time offset and the channel estimation process. As proposed in [11], first a set of possible time offsets is given, and for every offset a Channel Impulse Response (CIR) is estimated using the Least Square (LS) method. The estimated CIR allows the extraction of the LTF symbols. The correct time offset is then deduced by selecting the offset value that minimizes the Mean Square Error (MSE) criterion between the known LTF symbols and the estimated LTF symbols.

Compared to NDA algorithms, generally the performance of an IEEE 802.11a communication system using only training sequences (i.e., DA technique) is significantly improved. However the existing algorithms only exploit the preamble while the exploitation of any available redundancy (which is a main advantage of the NDA algorithm) has not been introduced.

An interesting combination of advantages of the NDA and DA algorithms is found in [12]. In this work, apart from the commonly used training sequence, the CTS also exploits the SIGNAL field of the IEEE 802.11a standard since its unknown parts are predictable via the Request to Send (RtS) control frame when the
Carrier Sense Multiple Access with Collision Avoidance (CSMA/CA) mechanism is activated. In addition, to improve the performance of the CTS step, a joint FTS and channel estimation based on the MinimumMean Squared-Error (MMSE) criterion is developed. Subsequently, in [13] a joint FTS and channel estimation performing the MAP criterion instead of the MMSE criterion is considered.

The results provided in [12] and [13], where the additional information (SIGNAL field) is exploited, motivates us to further investigate this direction. We propose a new time and frequency synchronization algorithm for IEEE 802.11a communication system where the SIGNAL field is extensively exploited by the algorithm at several levels. Moreover, the channel information, provided from the reception of the RtS control frame, is used for improving the accuracy of the CTS stage, as compared to that in [12] and [13].

This paper is organized as follows. The next section describes the communication system in accordance to the IEEE 802.11a standard to better take into account the structure of the physical frame. Section 3 reviews some important synchronization algorithms. Section 4 concerns the proposed time and frequency synchronisation algorithm. Section 5 provides and discusses the simulation results. Section 6 concludes the work.

\section{IEEE 802.11A Physical PACKet}

The IEEE 802.11a physical packet is composed of three fields: a PREAMBLE training field, a SIGNAL field and a DATA field (see Figure 1). The PREAMBLE field helps the receiving station to synchronize with respect to the transmitting station. This field is composed of: (i) ten identical STF, usually used for Automation Gain Control (AGC), diversity selection, signal detect and Coarse Frequency Synchronization (CFS); and (ii) two identical LTF, reserved for the channel estimation and Fine Frequency Synchronization (FFS). The SIGNAL field provides information about the transmission rate (in Mbits/s) and the length of the DATA field (in octets).

The physical packet modulation follows the specifications of the IEEE 802.11a wireless communication system as summarized in Figure 2. The binary SIGNAL sequence is used as an input of the convolutional encoder with a constraint length $K=7$, a polynomial generator $[171,133]$, and a code rate $R=1 / 2$. Its outputs are then interleaved (by a known interleaver) and then BPSK (Binary Phase Shift Keying) modulated. This is followed by pilot insertion and OFDM modulation, with an $N$-point Inverse Fast Fourier Transform (IFFT). Modulated similarly to the SIGNAL field, however the DATA field bits are applied to a scrambler before the convolutional encoder. Furthermore variable code rates (with $R=1 / 2,2 / 3$ or $3 / 4$ ) and different modulations (e.g. M-PSK or M-QAM) are available depending on the desired data rate to achieve.

The IFFT is applied to the symbols $X(k)$, for $0 \leq$ $k \leq N-1$, to get samples $x(n)$ in the time domain. 


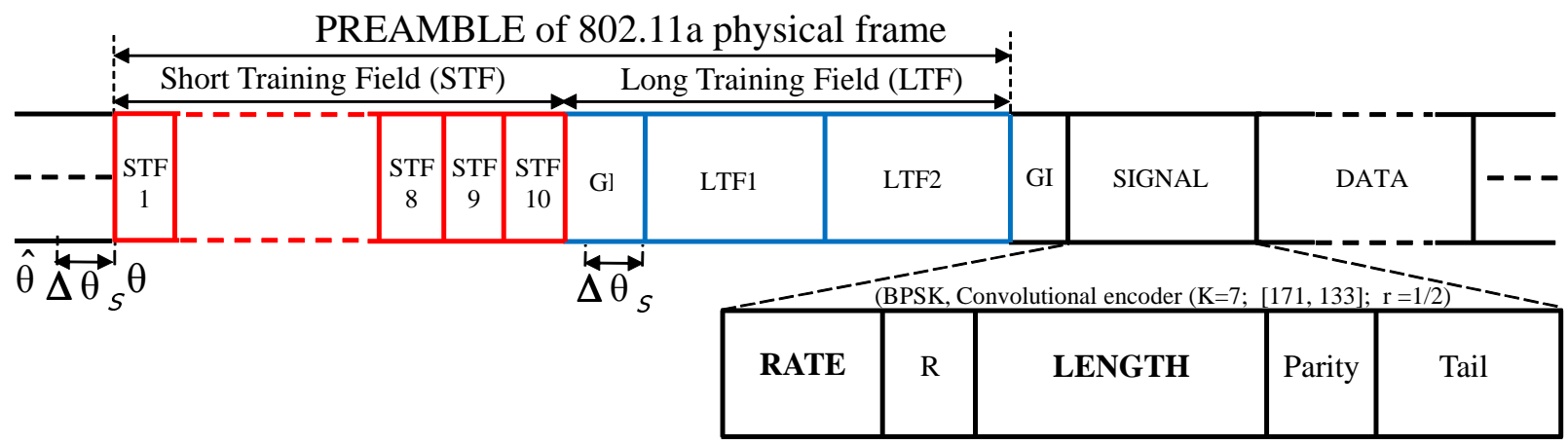

Figure 1. IEEE 802.11a physical packet.

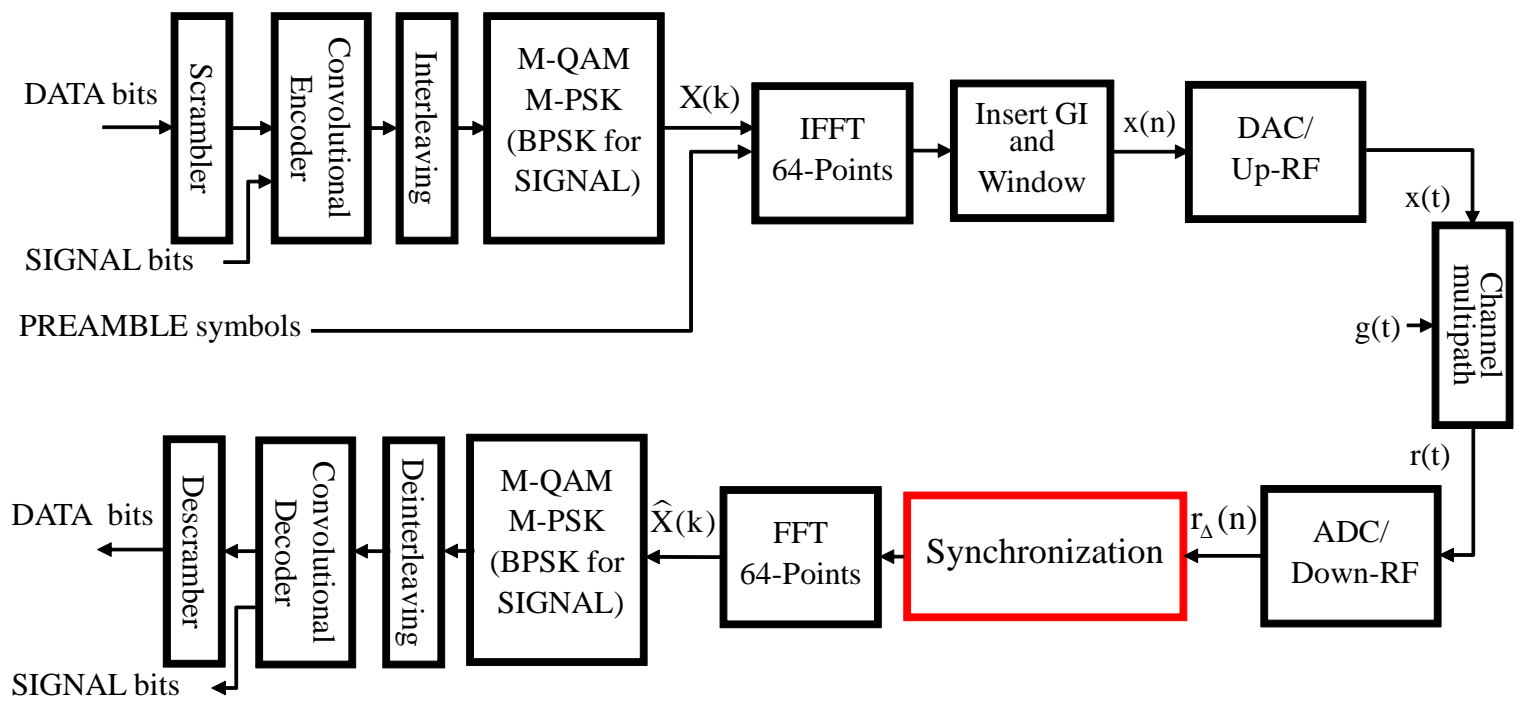

Figure 2. Wireless communication system using OFDM.

Note that in the 802.11a standard the number $N$ of IFFT points is also the length of one LTF repetition. Then, each OFDM symbol is generated from these samples and preceded by a Cyclic Prefix (CP). The samples $x(n)$ are then shaped by a specific window prior to being transmitted via a multipath fading channel described by a Finite Impulse Response (FIR) filter of length $L$. At the receiver, the received discrete baseband signal $r_{\Delta}(n)$ is expressed by

$$
r_{\Delta}(n)=\sum_{i=0}^{L-1} h(i) x(n-i-\theta) e^{j 2 \pi \epsilon(n-\theta) / N}+g(n),
$$

where $h(i)$ is the slowly time-varying discrete complex Channel Impulse Response (CIR) with $\sum_{i=0}^{L-1} \mathrm{E}\left\{|h(i)|^{2}\right\}=1$ (E is the expectation operator), $L$ is the number of channel taps, $g(n)$ is the complex Additive White Gaussian Noise (AWGN), $\epsilon=\Delta F_{c} T$ is the normalized frequency offset with $\Delta F_{c}$ being the frequency offset between the transmitter and the receiver, $T$ being the OFDM symbol duration, and $\theta$ is the symbol timing. In order to correctly demodulate $r_{\Delta}(n)$, time synchronization and frequency synchronization are required to estimate $\theta$ and $\epsilon$ respectively. This is the goal of this paper.

\section{StATE-OF-THE-ARTS ON SyNCHRONIZATION}

Among the synchronization algorithms developed in the literature, this section has selected two kinds of algorithms, which will be briefly described. One is related to redundant information (NDA) [14, 15] and the other on training sequences (DA) [8-10].

In [14], the CP of the OFDM symbol was exploited. The symbol timing is estimated by searching the index that provides the minimum difference between the two sliding windows as

$$
\widehat{\theta}=\arg \min _{\theta} \sum_{n=0}^{N_{g}-1}\left|r_{\Delta}(n+\theta)-r_{\Delta}(n+\theta+N)\right|,
$$

where $N_{g}$ the length of the $\mathrm{CP}$ and $N$ the length of the actual OFDM symbol.

If $\mathrm{CFO}$ exists, the solution provided by Equation (2) may be inaccurate. To deal with this situation, the authors of [15] proposed to minimize the squared difference between the received signal corresponding to the first sliding window and the conjugate received signal associated to the second sliding window. Accordingly, the symbol timing is given by

$$
\widehat{\theta}=\arg \min _{\theta} \sum_{n=0}^{N_{g}-1}\left(\left|r_{\Delta}(n+\theta)\right|-\left|r_{\Delta}^{*}(n+\theta+N)\right|\right)^{2} .
$$


The symbol timing can also be deduced from the index which maximizes the ACF as follows:

$$
\widehat{\theta}=\arg \max _{\theta} \sum_{n=0}^{N_{g}-1} r_{\Delta}(n+\theta) r_{\Delta}^{*}(n+\theta+N) .
$$

The DA algorithms developed in [9] and [10] not only have low computational complexity but also are adapted to the IEEE 802.11a standard. To estimate the time offset, these algorithms proceed in two main steps: the Coarse Time Synchronization (CTS) step followed by the Fine Time Synchronization (FTS) step to estimate the remaining time offset.

The CTS is based on the normalized ACF

$$
\bar{R}(\theta)=\frac{\sum_{n=0}^{143} r_{\Delta}^{*}(n+\theta) r_{\Delta}(n+\theta+16)}{\sum_{n=0}^{143}\left|r_{\Delta}(n+\theta)\right|^{2}},
$$

applied on the STF (i.e., 160 samples) as specified by the standard, and the symbol timing is the one which maximizes the normalized ACT function, and is given by

$$
\widehat{\theta}=\arg \max _{\theta} \bar{R}(\theta) .
$$

In [10], the CFO is then estimated as

$$
\widehat{\epsilon}_{\mathrm{CFO}}=\frac{N}{2 \pi 16} \angle \sum_{n=0}^{143} r_{\Delta}^{*}(n+\hat{\theta}) r_{\Delta}(n+\hat{\theta}+16) .
$$

To improve the frequency synchronization accuracy provided by (6), the authors of [9] proposed to adjust the CFO with FFS step which is deduced from the ACF applied on the LTF repetitions as follows:

$$
\widehat{\epsilon}_{\mathrm{FFO}}=\frac{N}{2 \pi 64} \angle \sum_{n=0}^{63} r_{\Delta}^{*}(n+\hat{\theta}+192) r_{\Delta}(n+\hat{\theta}+256) .
$$

The estimated CFO is then deduced to

$$
\hat{\epsilon}=\widehat{\epsilon}_{\mathrm{CFO}}+\widehat{\epsilon}_{\mathrm{FFO}},
$$

and is used for compensating the received signal.

Following these operations, FTS is now considered. Denote $r^{\prime}(n)$ the received signal which is compensated after CTS and frequency synchronization steps. The estimated remaining time offset $\Delta \widehat{\theta}$ is one of the possible positions in the set $\Lambda$ maximizing the CCF between $r^{\prime}(n)$ and a part of the known LTF denoted $g_{L T F}(n)$ (first 32 over 128 samples) and is thus given by

$$
\Delta \widehat{\theta}=\arg \max _{\Delta \theta \in \Lambda}\left|\sum_{n=0}^{31} g_{\mathrm{LTF}}^{*}(n) r^{\prime}(n+\Delta \theta)\right|^{2} .
$$

In [8], to improve the performance of the CFO estimation, a frequency synchronization algorithm (FSbased MAP) was developed where a transmission burst consisting of some pilot OFDM symbols is employed. The time offset is assumed to be perfectly compensated (i.e., $\theta=0$ ). For convenience, the received signal corresponding to one pilot OFDM symbol of length $N$ is expressed in matrix form as

$$
\mathbf{r}^{\mathrm{p}}=\boldsymbol{\Phi}_{\epsilon}^{\mathrm{p}} \mathbf{S}^{\mathrm{p}} \mathbf{h}+\mathbf{g},
$$

where

$$
\begin{aligned}
\mathbf{r}^{\mathrm{p}} & =[r(n), r(n+1), \ldots, r(n+N-1)]^{T}, \\
\mathbf{\Phi}_{\epsilon}^{\mathrm{p}} & =\operatorname{diag}\left\{e^{j 2 \pi \epsilon n / N}, e^{j 2 \pi \epsilon(n+1) / N}, \ldots, e^{j 2 \pi \epsilon(n+N-1) / N}\right\}, \\
\mathbf{S}^{\mathrm{p}} & =\left[\mathbf{S}_{0}^{\mathrm{p}}, \mathbf{S}_{1}^{\mathrm{p}}, \ldots, \mathbf{S}_{L-1}^{\mathrm{p}}\right], \\
\mathbf{S}_{l}^{\mathrm{p}} & =[x(n-l), x(n+1-l), \ldots, x(n+N-1-l)]^{T}, \\
\mathbf{h} & =[h(0), h(1), \ldots, h(L-1)]^{T}, \\
\mathbf{g} & =[g(n), g(n+1), \ldots, g(n+N-1)]^{T}
\end{aligned}
$$

In the above, the superscript " $\mathrm{p}$ " indicates "pilot", $r(n)$ is given by Equation (1) with $\theta=0$, and $x(n)$ is the known pilot sample in the time domain with $l=0, \ldots, L-1$.

The MAP estimates of the normalized frequency offset and channel are given by

$$
\{\hat{\mathbf{h}}, \hat{\epsilon}\}=\arg \max _{\mathbf{h}, \epsilon} \ln P\left(\mathbf{h}, \epsilon \mid \mathbf{r}^{\mathrm{p}}\right)
$$

where $P$ is the a posteriori probability density function of $\mathbf{h}$ and $\epsilon$ given $\mathbf{r}^{\mathrm{p}}$. Calculating $P\left(\mathbf{h}, \epsilon \mid \mathbf{r}^{\mathrm{p}}\right)$ under the assumption that $\epsilon$ is uniformly distributed in the range $\left[-\epsilon_{0}, \epsilon_{0}\right]$, the MAP estimates of CFO and channel coefficients are

$$
\{\hat{\mathbf{h}}, \hat{\epsilon}\}=\arg \min _{\mathbf{h}, \epsilon} f_{\mathrm{MAP}}(\mathbf{h}, \epsilon),
$$

where

$$
f_{\mathrm{MAP}}(\mathbf{h}, \epsilon)=\frac{1}{\sigma_{g}^{2}}\left\|\mathbf{r}^{\mathrm{p}}-\mathbf{\Phi}_{\epsilon}^{\mathrm{p}} \mathbf{S}^{\mathrm{p}} \mathbf{h}\right\|^{2}+\mathbf{h}^{\mathrm{H}} \mathbf{R}_{\mathbf{h}}^{-\mathbf{1}} \mathbf{h},
$$

with $\sigma_{g}^{2}$ and $\mathbf{R}_{\mathbf{h}}$ being the noise variance and the channel covariance matrix (for more details, see $[8$, Section IV]). Estimating the gradient vector of $f_{\mathrm{MAP}}(\mathbf{h}, \boldsymbol{\epsilon})$ with respect to $\mathbf{h}^{H}$ and setting it to zero to obtain the following MAP estimate of the channel:

$$
\widehat{\mathbf{h}}=\left(\left(\mathbf{S}^{\mathrm{p}}\right)^{H} \mathbf{S}^{\mathrm{p}}+\sigma_{g}^{2} \mathbf{R}_{\mathbf{h}}^{-\mathbf{1}}\right)^{-1}\left(\mathbf{S}^{\mathrm{p}}\right)^{H}\left(\boldsymbol{\Phi}_{\epsilon}^{\mathrm{p}}\right)^{H} \mathbf{r}^{\mathrm{p}} .
$$

Replacing (13) into $f_{\mathrm{MAP}}(\mathbf{h}, \epsilon)$ provides the $\mathrm{CFO}$ estimate

$$
\hat{\epsilon}=\arg \min _{\epsilon} g_{\mathrm{MAP}}(\epsilon),
$$

where

$$
\begin{aligned}
g_{\mathrm{MAP}}(\boldsymbol{\epsilon}) & =\left(\mathbf{r}^{\mathrm{p}}\right)^{H} \boldsymbol{\Phi}_{\epsilon}^{\mathrm{p}}\left(\mathbf{S}^{\mathrm{p}}\right)^{+}\left(\boldsymbol{\Phi}_{\epsilon}^{\mathrm{p}}\right)^{H} \mathbf{r}^{\mathrm{p}}, \\
\left(\mathbf{S}^{\mathrm{p}}\right)^{+} & =\mathbf{S}^{\mathrm{p}}\left[\left(\mathbf{S}^{\mathrm{p}}\right)^{H} \mathbf{S}^{\mathrm{p}}+\mathbf{R}_{\mathbf{h}}^{-\mathbf{1}} \sigma_{g}^{2}\right]^{-1}\left(\mathbf{S}^{\mathrm{p}}\right)^{H} .
\end{aligned}
$$

To determine the CFO estimate, the Newton-Raphson approximation is calculated as follows:

$$
\hat{\epsilon}_{i+1}=\hat{\epsilon}_{i}-\left.\left[\frac{\partial^{2} g(\epsilon)}{\partial \epsilon^{2}}\right]^{-1} \frac{\partial g(\epsilon)}{\partial \epsilon}\right|_{\hat{\epsilon}=\epsilon_{i}{ }^{\prime}}
$$

where $\hat{\epsilon}_{i}$ represents the CFO estimation at the $i^{\text {th }}$ iteration, and

$$
\begin{aligned}
& \frac{\partial g(\epsilon)}{\partial \epsilon}= 2 \Re\left\{\left(\mathbf{r}^{\mathrm{p}}\right)^{H} \mathbf{G}^{\mathrm{p}} \boldsymbol{\Phi}_{\epsilon}^{\mathrm{p}}\left(\mathbf{S}^{\mathrm{p}}\right)^{+}\left(\boldsymbol{\Phi}_{\epsilon}^{\mathrm{p}}\right)^{H} \mathbf{r}^{\mathrm{p}}\right\}, \\
& \frac{\partial g^{2}(\epsilon)}{\partial \epsilon^{2}}=2 \Re\left\{\left(\mathbf{r}^{\mathrm{p}}\right)^{H}\left(\mathbf{G}^{\mathrm{p}}\right)^{2} \boldsymbol{\Phi}_{\epsilon}^{\mathrm{p}}\left(\mathbf{S}^{\mathrm{p}}\right)^{+}\left(\Phi_{\epsilon}^{\mathrm{p}}\right)^{H} \mathbf{r}^{\mathrm{p}}+\right. \\
&\left.\left.\left(\mathbf{r}^{\mathrm{p}}\right)^{H} \mathbf{G}^{\mathrm{p}} \boldsymbol{\Phi}_{\epsilon}^{\mathrm{p}}\left(\mathbf{S}^{\mathrm{p}}\right)^{+}\left(\mathbf{G}^{\mathrm{p}}\right)^{H}\left(\boldsymbol{\Phi}_{\epsilon}^{\mathrm{p}}\right)^{H} \mathbf{r}^{\mathrm{p}}\right)\right\}, \\
& \mathbf{G}^{\mathrm{p}}=j \frac{2 \pi}{N} \operatorname{diag}\{n, n+1, \ldots, n+N-1\} .
\end{aligned}
$$




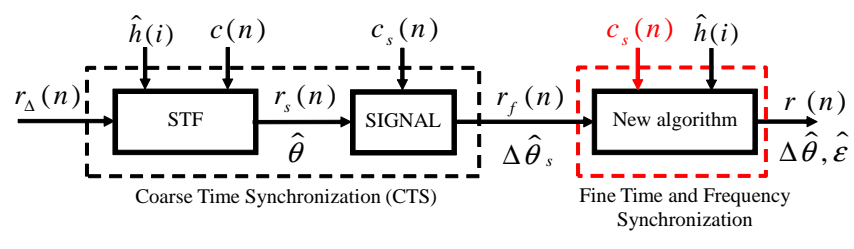

Figure 3. Proposed time and frequency synchronization algorithm.

\section{Proposed Synchronization Algorithm}

To improve the synchronization of stations in a wireless communication system, the underlying idea of our approach consists in extracting information at the IEEE 802.11a physical layer in order to be exploited by the receiver as known information, in addition to the usuel training sequences (i.e., STF and LTF). Our investigations allow the identification the SIGNAL field when the CSMA/CA mechanism is activated to avoid collisions in the wireless communication system. Indeed the parts of the SIGNAL field are either known or predictable from the RtS control frame when the CSMA/CA mechanism is triggered jointly with bit-rate adaptation algorithms to the channel.

The proposed synchronisation algorithm, as summarized in Figure 3, is performed by the receiver in three main stages: (i) extracting the redundant information to determine the SIGNAL field (see Section 4.1); (ii) coarse time synchronization using the SIGNAL field and channel estimation from the RtS control frame (see Section 4.2); and (iii) joint fine time synchronization and frequency offset estimation (see Section 4.3).

\subsection{Characterization of the SIGNAL field}

The objective of this section is to identify the two main parts of the SIGNAL field (see Figure1), which are "RATE" and "LENGTH". The transmitter initiates the CSMA/CA mechanism by sending a RtS control frame to ask the receiver if it is available [16] (see Figure 4). If it is the case, the receiver performs a rate adaptation algorithm by measuring the Signal to Noise Ratio (SNR) level of the received RtS frame to estimate the channel conditions [17]. Then it replies with a Clear to Send (CtS) control frame to: (i) inform other stations of its unavailability to receive information coming from other stations during a specified period of time; and (ii) suggest to the transmitter a transmission rate that the sender should use to transmit its physical packet. By the way the receiver has a knowledge of the transmission rate corresponding to the value of the RATE subfield of the SIGNAL field. Therefore the unknown LENGTH subfield of the SIGNAL is deduced from the following relationship since the RATE value is known [1]:

$$
\begin{aligned}
& \text { LENGTH }=\text { RATE } \times \\
& \quad \frac{\left(T_{\text {packet }}-T_{\text {pre }}-T_{\text {SIGNAL }}-\left(T_{\text {symb }} / 2\right)\right)-22}{8},
\end{aligned}
$$

where $T_{\text {pre }}, T_{\text {SIGNAL }}$ and $T_{\text {symb }}$ are known durations (in micro-seconds) of the PREAMBLE, the SIGNAL field and the OFDM symbol, respectively referred in [1]. The

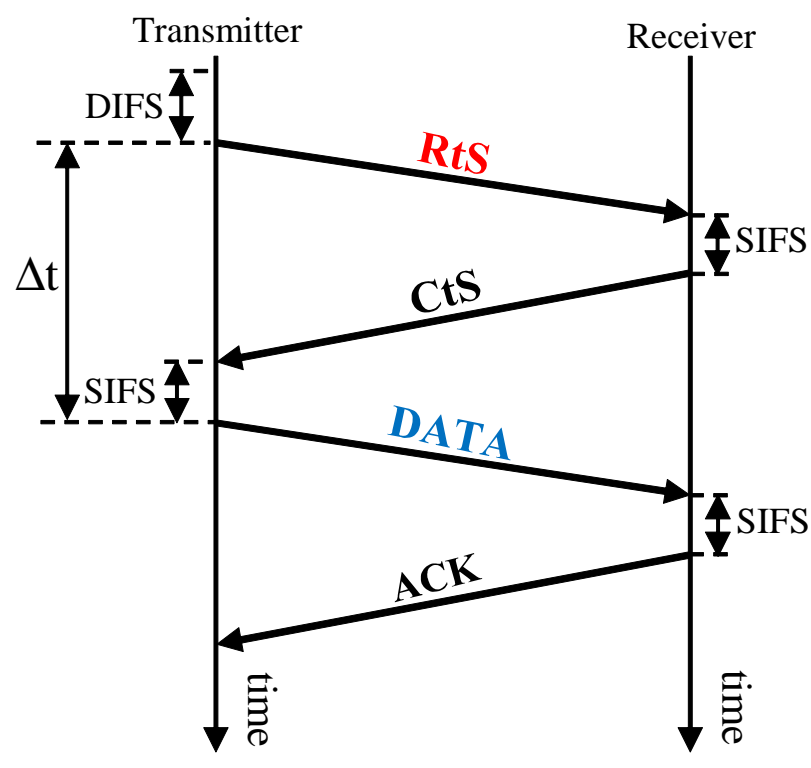

Figure 4. RtS/CtS handshake with active CSMA/CA mechanism.

$T_{\text {packet }}$ is the duration (in micro-seconds) required to transmit the DATA physical packet. This parameter can be deduced from the DURATION field value of the RtS frame which is given by [18]:

$$
\text { DURATION }=3 T_{\text {SIFS }}+T_{\mathrm{CtS}}+T_{\mathrm{ACK}}+T_{\text {packet }},
$$

where $T_{\text {SIFS }}$ is the known duration (in micro-seconds) of a Short Inter-Frame Space, $T_{\mathrm{CTS}}$ and $T_{\mathrm{ACK}}$ are respectively the known required durations to transmit $\mathrm{CtS}$ and Acknowledgement (ACK) frames. From the knowledge of the RATE, LENGTH and "R" (Reserved), the "Parity" field corresponding to one bit is deduced. With six zero tail bits appended, the 24-bits SIGNAL field is completely identified. The receiver thus exploits this field for synchronization.

\subsection{Coarse time synchronization (CTS)}

This section estimates the symbol timing of the received signal, which is $\theta$ in Equation (1). For this, two steps are performed and described below.

4.2.1 Symbol timing estimation: The receiver estimates the symbol timing using the CCF between the received signal $r_{\Delta}(n)$ and the known STF $c(n)$. However if the transmitted signal is heavily distorted by the wireless channel, the symbol timing estimation will be affected. Therefore, instead of directly using the received signal in the CCF, we propose to estimate the transmitted signal; this will improve the estimation accuracy as explained below.

To estimate the transmitted signal, $x(n)$, we start with the channel estimation based on the information knowledge extracted from the protocol when the CSMA/CA is activated. To allow the stations of any wireless system to receive the RtS control frame, the transmitting station has to send this frame with a power level higher than the nominal transmission power level at which the DATA frame is sent [19]. According to 
this strategy, we assume that the transmitter and receiver stations have been correctly synchronized during the medium reservation negotiation (i.e., $\mathrm{RtS} / \mathrm{CtS}$ ) to enable the transmission of the physical DATA packet. Moreover the channel is assumed to be static between the transmission duration of RtS and DATA frames (see Figure 4). Indeed under worst transmission/reception conditions of the $\mathrm{CtS}$ control frame the interval time between the transmitted physical packet and RtS control frame $\left(T_{\mathrm{RtS}}+T_{\mathrm{CtS}}+2 T_{\mathrm{SIFS}}\right)$ is small (e.g., $124 \mu \mathrm{s}$ with the lowest rate of $6 \mathrm{Mb} / \mathrm{s}$ ), meaning that the Doppler frequency can be considered as a small value.

Note that the PREAMBLE fields of both the control (e.g., RtS, CtS) and the DATA frames (see Figure 1) are the same. The channel estimation, as specified by the standard for the physical packet, is related to the LTF of the RtS PREAMBLE. A MAP-based channel estimation is then given by

$$
\widehat{\mathbf{h}}=\left(\mathbf{G}^{H} \mathbf{G}+\sigma_{g}^{2} \mathbf{R}_{\mathbf{h}}^{-\mathbf{1}}\right)^{-1}\left(\mathbf{G}^{H} \mathbf{r}_{R t S}+\sigma_{g}^{2} \mathbf{R}_{\mathbf{h}}^{-\mathbf{1}} \boldsymbol{\mu}_{h}\right),
$$

where $\mathbf{r}_{R t S}$ is the received RtS frame signal corresponding to the LTF sequence, G contains the LTF training samples, $\sigma_{g}^{2}$ is the noise variance, $\mathbf{R}_{\mathbf{h}}$ is the covariance matrix of the true channel, and $\mu_{h}$ is the mean vector of the true channel. Instead of using a Power Delay Profile (PDP) to calculate $\mathbf{R}_{\mathbf{h}}=E\left\{\mathbf{h} \mathbf{h}^{H}\right\}$, we propose to replace the true channel $\mathbf{h}$ by its Least-Square (LS) estimation $\tilde{\mathbf{h}}$ given by the IFFT of $\tilde{\mathbf{H}}=\mathbf{X}^{-1} \mathbf{R}_{\mathrm{RtS}}$ where $\mathbf{X}$ the diagonal matrix whose elements are the known LTF symbols and $\mathbf{R}_{\mathrm{RtS}}$ the received symbol vector.

Denote $\hat{H}(k)$ (with $0 \leq k \leq N-1$ ) the channel estimate and $R_{\Delta}(k)$ (see $((1))$ ) the received symbols corresponding to the DATA frame in the frequency domain, then the transmitted symbol estimate, $\hat{X}(k)$, obtained by using a Zero-Forcing (ZF) equalizer, is given by

$$
\hat{X}(k)=\frac{R_{\Delta}(k)}{\hat{H}(k)},
$$

and the time-domain estimate of the transmitted signal is

$$
\hat{x}(n)=\frac{1}{N} \sum_{i=0}^{N-1} \hat{X}(k) e^{j 2 \pi k n / N}
$$

The symbol timing is then deduced as the index of the maximum value of CCF between the estimated transmitted signal $\hat{x}(n)$ and the known STF $c(n)$ of length $L_{\mathrm{STF}}$, which is given by

$$
\hat{\theta}=\arg \max _{\theta} \sum_{n=0}^{L_{\mathrm{STF}}-1} c^{*}(n) \hat{x}(n+\theta) .
$$

4.2.2 CTS using the SIGNAL field: The received signal still affected by the remaining time offset (i.e., $\Delta \theta_{s}=$ $\hat{\theta}-\theta)$ is expressed by

$$
r_{S}(n)=\sum_{i=0}^{L-1} h(i) x\left(n-i-\Delta \theta_{s}\right) e^{j 2 \pi \epsilon\left(n-\Delta \theta_{s}\right) / N}+g(n) .
$$

To estimate $\Delta \theta_{s}$, the 802.11 a SIGNAL field (see Figure 1) was exploited in $[12,13]$ as a new additional training sequence at the receiver since all parts of this field are completely known (see Equations (16) and (17)).
The CCF is then performed between the known SIGNAL field $c_{S}(n)$ of length $L_{\text {SIG }}$ (i.e., CP length added to the SIGNAL length) and the received signal $r_{S}(n)$. The remaining time offset is then deduced from the index, among the set of possible values $\Theta=\left\{\Delta \theta_{s}^{(k)} \mid k=\right.$ $-K, \ldots, K ; K \in \mathbf{N}\}$, which maximizes the CCF as given by

$$
\Delta \widehat{\theta_{S}}=\arg \max _{\Delta \theta_{S}^{(k)} \in \Theta} \sum_{n=0}^{L_{S I G}-1} c_{S}^{*}(n) r_{\mathcal{S}}\left(n+\Delta \theta_{S}^{(k)}\right) .
$$

After this step, the received signal with a remaining time offset $\Delta \theta$ (i.e., $\Delta \theta=\Delta \widehat{\theta_{S}}-\Delta \theta_{S}$ ) is expressed by

$$
r_{f}(n)=\sum_{i=0}^{L-1} h(i) x(n-i-\Delta \theta) e^{j 2 \pi \epsilon(n-\Delta \theta) / N}+g(n) \text {. }
$$

\subsection{Joint MAP frequency synchronization and channel estimation using the SIGNAL field}

This section proposes to estimate the remaining time offset $\Delta \theta$ and the normalized frequency offset $\epsilon$. We develop a joint fine time and frequency synchronization based on the MAP criterion. To do so, we not only adapt the frequency synchronization algorithm (FSbased MAP) developed in [8] to the IEEE 802.11a specifications but also make changes to the algorithm since the authors assumed that the time synchronization is perfectly compensated. Knowledge of the SIGNAL field is also taken into account in this stage as described below. The received signal $\mathbf{r}$ corresponding to the two LTF repetitions and the SIGNAL field is expressed in a matrix form as follows:

$$
\mathbf{r}=\boldsymbol{\Phi}_{\Delta \theta, \epsilon} \mathbf{S}_{\Delta \theta} \mathbf{h}+\mathbf{g},
$$

where

$$
\begin{aligned}
\mathbf{r}= & {\left[r_{f}(n), \ldots, r_{f}\left(n+2 N+N_{G}+N_{S}-1\right)\right]^{T}, } \\
\mathbf{S}_{\Delta \theta}= & {\left[\mathbf{S}_{0, \Delta \theta}, \mathbf{S}_{1, \Delta \theta}, \ldots, \mathbf{S}_{L-1, \Delta \theta}\right], } \\
\mathbf{S}_{l, \Delta \theta}= & {[x(n-l-\Delta \theta), x(n+1-l-\Delta \theta), \ldots,} \\
& \left.x\left(n+2 N+N_{G}+N_{S}-1-l-\Delta \theta\right)\right]^{T}, \\
\mathbf{h}=[h(0), h(1), \ldots, h(L-1)]^{T},(C I R) & \mathbf{g}=[g(n-\Delta \theta), g(n-\Delta \theta+1), \ldots, \\
\mathbf{\Phi}_{\Delta \theta, \epsilon}= & \operatorname{diag}\left\{e^{j \frac{2 \pi \epsilon(n-\Delta \theta)}{N}}, \ldots, e^{j \frac{2 \pi \epsilon\left(n-\Delta \theta+2 N+N_{S}+N_{G}-1\right)}{N}}\right\} .
\end{aligned}
$$

Above, $x(n)$ is the known LTF and SIGNAL sample in the time domain $(0 \leq l \leq L-1), N$ the number of samples of one LTF repetition, $N_{S}$ the length of the SIGNAL field and $N_{G}$ the length of its guard interval, $\mathbf{h}$ and $\mathbf{g}$ are the CIR and noise vectors.

The remaining time offset $\Delta \theta$, the normalized frequency offset $\epsilon$ and the CIR $\mathbf{h}$ are jointly estimated according to the MAP criterion as follows:

$$
\{\hat{\mathbf{h}}, \Delta \widehat{\theta}, \hat{\epsilon}\}=\arg \max _{\mathbf{h}, \Delta \theta, \epsilon} \ln P(\mathbf{h}, \Delta \theta, \epsilon \mid \mathbf{r}),
$$

where $P$ is the a posteriori probability density function of $\mathbf{h}, \Delta \theta$ and $\epsilon$ given $\mathbf{r}$. 
In this section, $\epsilon$ is also assumed to be uniformly distributed in the range $\left[-\epsilon_{0}, \epsilon_{0}\right]$. To realize $(26)$, we define a set $\Lambda$ containing $2 M+1$ possible time offset values; $\boldsymbol{\Lambda}=\left\{-\Delta \theta_{M}, \ldots, \Delta \theta_{M}\right\}$. For a given value $\Delta \theta_{m} \in$ $\Lambda$, the MAP-based estimates of the CFO and channel coefficients correspondingly are

$$
\left\{\hat{\mathbf{h}}_{\Delta \theta_{m}}, \hat{\epsilon}_{\Delta \theta_{m}}\right\}=\arg \min _{\mathbf{h}, \epsilon} f_{\mathrm{MAP}}^{(m)}(\mathbf{h}, \epsilon),
$$

where

$$
f_{\mathrm{MAP}}^{(m)}(\mathbf{h}, \epsilon)=\frac{1}{\sigma_{g}^{2}}\left\|\mathbf{r}_{\Delta \theta_{m}}-\boldsymbol{\Phi}_{\Delta \theta_{m}, \epsilon} \mathbf{S}_{\Delta \theta_{m}} \mathbf{h}\right\|^{2}+\mathbf{h}^{\mathbf{H}} \mathbf{R}_{\mathbf{h}}^{-\mathbf{1}} \mathbf{h},
$$

with $\mathbf{r}_{\Delta \theta_{m}}$ being the received signal corresponding to the offset value $\Delta \theta_{m}$. Setting the gradient vector of $f_{\text {MAP }}^{(m)}(\mathbf{h}, \epsilon)$ with respect to $\mathbf{h}^{H}$ to zero provides the MAP-based channel estimate

$$
\widehat{\mathbf{h}}_{\Delta \theta_{m}}=\left[\mathbf{S}_{\Delta \theta_{m}}^{H} \mathbf{S}_{\Delta \theta_{m}}+\sigma_{g}^{2} \mathbf{R}_{\mathbf{h}}^{-\mathbf{1}}\right]^{-1} \mathbf{S}_{\Delta \theta_{m}}^{H} \boldsymbol{\Phi}_{\Delta \theta_{m}, \epsilon}^{H} \mathbf{r}_{\Delta \theta_{m}} .
$$

Replacing (28) into $f_{\mathrm{MAP}}^{(m)}(\mathbf{h}, \epsilon)$ provides the CFO estimate

$$
\hat{\epsilon}_{\Delta \theta_{m}}=\arg \min _{\epsilon} g_{\mathrm{MAP}}^{(m)}(\epsilon),
$$

where

$$
\begin{aligned}
g_{\mathrm{MAP}}^{(m)}(\epsilon) & =\mathbf{r}_{\Delta \theta_{m}}^{H} \boldsymbol{\Phi}_{\Delta \theta_{m}, \epsilon} \mathbf{S}_{\Delta \theta_{m}}^{+} \boldsymbol{\Phi}_{\Delta \theta_{m}, \epsilon}^{H} \mathbf{r}_{\Delta \theta_{m} \prime} \\
\mathbf{S}_{\Delta \theta_{m}}^{+} & =\mathbf{S}_{\Delta \theta_{m}}\left[\mathbf{S}_{\Delta \theta_{m}}^{H} \mathbf{S}_{\Delta \theta_{m}}+\mathbf{R}_{\mathbf{h}}^{-\mathbf{1}} \sigma_{g}^{2}\right]^{-1} \mathbf{S}_{\Delta \theta_{m}}^{H} .
\end{aligned}
$$

The Newton-Raphson approximation is then calculated as follows:

$$
\hat{\epsilon}_{\Delta \theta_{m}, i+1}=\hat{\epsilon}_{\Delta \theta_{m}, i}-\left.\left[\frac{\partial^{2} g_{\mathrm{MAP}}^{(m)}(\epsilon)}{\partial \epsilon^{2}}\right]^{-1} \frac{\partial g_{\mathrm{MAP}}^{(m)}(\epsilon)}{\partial \epsilon}\right|_{\hat{\epsilon}=\epsilon_{i}}
$$

where $\hat{\epsilon}_{\Delta \theta_{m}, i}$ indicates the CFO estimation at the $i^{\text {th }}$ iteration, and

$$
\begin{gathered}
\frac{\partial g_{\mathrm{MAP}}^{(m)}(\epsilon)}{\partial \epsilon}=2 \Re\left\{\mathbf{r}_{\Delta \theta_{m}}^{H} \mathbf{G}_{\Delta \theta_{m}} \boldsymbol{\Phi}_{\Delta \theta_{m}, \epsilon} \mathbf{S}_{\Delta \theta_{m}}^{+} \boldsymbol{\Phi}_{\Delta \theta_{m}, \epsilon}^{H} \mathbf{r}_{\Delta \theta_{m}}\right\}, \\
\frac{\partial g_{\mathrm{MAP}}^{2}(\epsilon)}{\partial \epsilon^{2}}=2 \Re\left\{\mathbf{r}_{\Delta \theta_{m}}^{H} \mathbf{G}_{\Delta \theta_{m}}^{2} \boldsymbol{\Phi}_{\Delta \theta_{m}, \epsilon} \mathbf{S}_{\Delta \theta_{m}}^{+} \boldsymbol{\Phi}_{\Delta \theta_{m}, \epsilon}^{H} \mathbf{r}_{\Delta \theta_{m}}+\right. \\
\left.\mathbf{r}_{\Delta \theta_{m}}^{H} \mathbf{G}_{\Delta \theta_{m}} \boldsymbol{\Phi}_{\Delta \theta_{m}, \epsilon} \mathbf{S}_{\Delta \theta_{m}}^{+} \mathbf{G}_{\Delta \theta_{m}}^{H} \boldsymbol{\Phi}_{\Delta \theta_{m}, \epsilon}^{H} \mathbf{r}_{\Delta \theta_{m}}\right\}, \\
\mathbf{G}_{\Delta \theta_{m}}=j \frac{2 \pi}{N} \operatorname{diag}\left\{n-\Delta \theta_{m}, n-\Delta \theta_{m}+1, \ldots,\right. \\
\left.n-\Delta \theta_{m}+2 N+N_{S}+N_{G}-1\right\} .
\end{gathered}
$$

From (28) and (29), the CIR estimate is obtained by

$$
\widehat{\mathbf{h}}_{\Delta \theta_{m}}=\left[\mathbf{S}_{\Delta \theta_{m}}^{H} \mathbf{S}_{\Delta \theta_{m}}+\sigma_{g}^{2} \mathbf{R}_{\mathbf{h}}^{-\mathbf{1}}\right]^{-1} \mathbf{S}_{\Delta \theta_{m}}^{H} \boldsymbol{\Phi}_{\Delta \theta_{m}, \hat{\epsilon}_{m}}^{H} \mathbf{r}_{\Delta \theta_{m}} .
$$

Among the $2 M+1$ estimates of $\widehat{\mathbf{h}}_{\Delta \theta_{m}}$ based on (28), we select those that satisfy the following conditions:

$$
\left|\hat{h}_{\Delta \theta_{m}}(0)\right|>\beta \max _{\Delta \theta_{i}}\left|\hat{h}_{\Delta \theta_{i}}(0)\right|,
$$

where $\beta$ is a given threshold. Therefore, the set $\Lambda$ becomes $\Gamma$

$$
\Gamma=\left\{\omega_{0}, \ldots, \omega_{M^{\prime}} ; \quad M^{\prime} \leq 2 M\right\} .
$$

Finally, the remaining time offset is estimated by

$$
\Delta \widehat{\theta}=\arg \max _{\omega_{m^{\prime}}} \sum_{n=0}^{L-1}\left|\hat{h}_{\omega_{m^{\prime}}}(n)\right|^{2}
$$

\section{Simulation RESUlts}

This section discusses the performance of the proposed synchronization algorithm. Table I lists the simulation parameters as specified by the IEEE 802.11a standard in presence of multipath channel COST207-RA which follows the Rice model with a Line-Of-Sight (LOS) [1]. According to the standard, the tolerance of the in-

Table I

Simulation Parameters

\begin{tabular}{ll}
\hline \hline Parameters & Values \\
\hline Bandwidth $(B)$ & $20 \mathrm{MHz}$ \\
Sampling time $\left(T_{s}\right)$ & $50 \mathrm{~ns}$ \\
Number of subcarriers $\left(N_{c}\right)$ & 52 \\
Number of points FFT /IFT & 64 \\
Subcarrier spacing $(\Delta F)$ & $0.3125 \mathrm{MHz}$ \\
Channel model & Rice with COST207-RA \\
Channel time delay & $(0,200,400,600) \mathrm{ns}$ \\
Power of channel paths $\left(P_{c}\right)$ & $(0,-2,-10,-20) \mathrm{dB}$ \\
LOS & $(0, \mathrm{sqr}(0.91 / 0.41), 0.7)$ \\
Data rate & $6 \mathrm{Mbps}$ \\
$L_{\text {STF }}$ & 160 \\
$L_{\text {SIG }}$ & 80 \\
$K$ & 80 \\
\hline
\end{tabular}

ternal oscillator at each station belongs to the range $[-20,20]$ ppm and thus the total tolerance of the two stations falls in $[-40,40] \mathrm{ppm}$. For the carrier frequency $f_{c}=5.2 \mathrm{GHz}$ and the OFDM symbol duration $T=$ $N \times T_{S}=3.2 \mu s$, the normalized frequency offset $\epsilon$ is taken randomly according to a uniform distribution from the range $[-0.6,0.6]$. Note that the symbol timing offset is also randomly distributed according to a uniform distribution. The performance of the following algorithms is compared:

i) Algorithm 1 [10] has been described in Section 3. The ACF relied on the STF is applied for the CTS and FS. The CCF based on the LTF is then employed for the FTS;

ii) Algorithm 1 with a perfect TS is Algorithm 1 but when the true symbol timing $\theta$ is known and is used for a perfect time synchronization;

iii) Algorithm 1 with a perfect FS is Algorithm 1 but when the true value of the frequency offset $\epsilon$ is known and is used for a perfect frequency synchronisation;

iv) Algorithm 2 is the algorithm presented in Section 4;

v) Algorithm 2 with a perfect TS is Algorithm 2 but when the true symbol timing $\theta$ is known and is used for a perfect time synchronization;

vi) Algorithm 2 with a perfect FS is Algorithm 2 but when the true frequency offset $\epsilon$ is known and perfectly compensated; that is, the received signal after coarse time synchronization stage is multiplied by $e^{-j 2 \pi \epsilon n / N}$. 


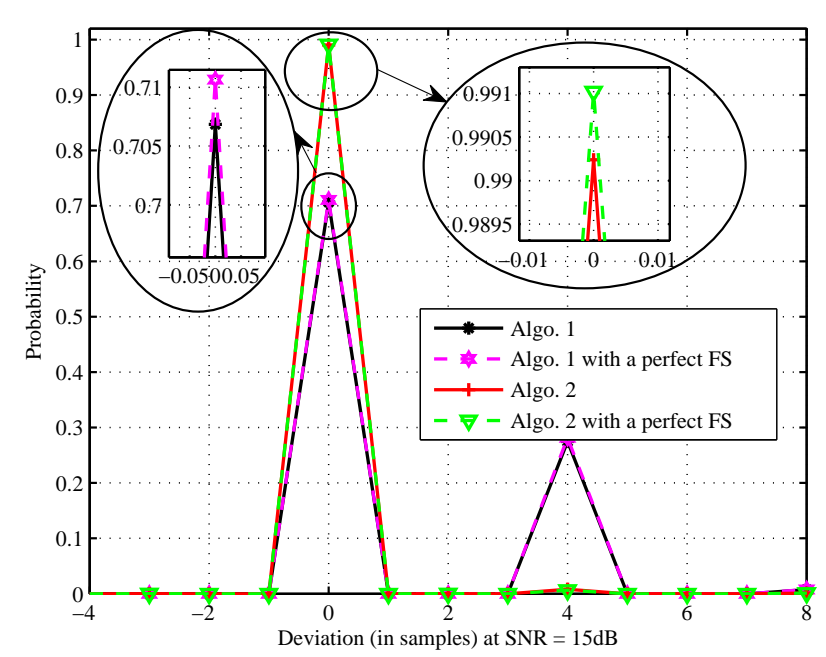

Figure 5. Deviation with respect to the true time position of a physical packet.

Figure 5 measures the detection probability of arrival time of the transmitted physical packet for a given deviation with respect to its true time position (i.e., $\widehat{\theta}-\theta)$ at $\mathrm{SNR}=15 \mathrm{~dB}$ for Algorithms 1 and 2 using $7.10^{4}$ test physical packets. Algorithm 2 provides the highest estimation accuracy (equal to $99 \%$ ) when the packet arrival time is detected without deviation compared to other algorithms. Its deviation interval is reduced to $[0,4]$ samples when the respective deviations of 1 and 2 are equal to zero. It is also possible to accept packets, the arrival time of which is estimated after the true position with a delay time less than 4 samples if we consider the $\widehat{\theta}_{\text {new }}=\widehat{\theta}-4$. This timing delay is accepted since the $\mathrm{CP}$ of the OFDM symbol and the maximum delay of the channel response are respectively equal to 16 and 13 samples and moreover the average power of tap 0 is one hundred than the one of tap 12 (i.e., $\left.P_{\text {ave }}(0)=100 \times P_{\text {ave }}(12)\right)$. In this case, the orthogonality of subcarrier frequency components is completely preserved in spite of the fact that there exists a phase offset which is however compensated by a single-tap frequency-domain equalizer.

The curves of Figure 6 illustrate the MSE (Mean Square Error) between the true $\mathrm{CFO}$ and its estimate $\left(E\left\{\left(\epsilon-\hat{\epsilon}_{m}\right)^{2}\right\}\right)$ versus Signal to Noise Ratio (SNR). The result analysis shows that regardless of the time synchronization being perfect or not, the MSE of our method is much lower than that of Algorithm 1. Indeed, at SNR=17.5 dB, MSE(Algo.1) $=4.2 \times 10^{-5}$ and MSE(Algo.2) $=1.6 \times 10^{-6}$. Moreover the two curves corresponding to Algorithm 2 are similar showing that even if the time offset is estimated the MSE is not affected compared to a perfect time offset compensation.

Figure 7 provides the PSF (Probability of Synchronization Failure) versus SNR. At SNR $=17.5 \mathrm{~dB}$ and with no deviation, the PSF of Algorithms 1 and 2 are as follows: PSF(Algo.1, perfect FS) = $2.5 \times 10^{-1}, \mathrm{PSF}\left(\right.$ Algo.2, perfect FS) $=7.2 \times 10^{-3}$ and PSF(Algo.2) $=8.7 \times 10^{-3}$ which is close to the PSF calculated when the frequency offset is perfectly compen-

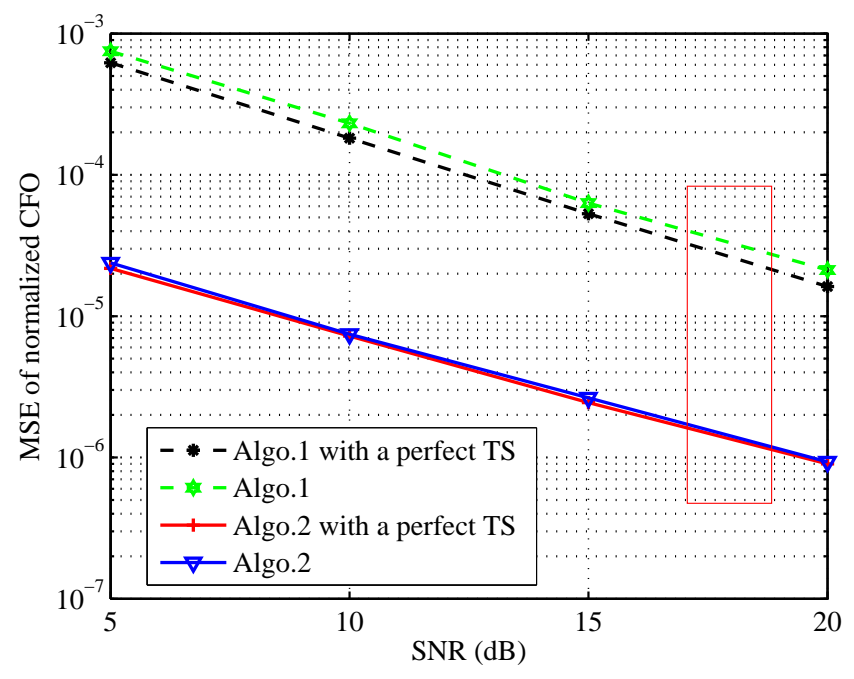

Figure 6. MSE of normalized frequency offset (the rectangular box presents the operating area of the 802.11a standard).

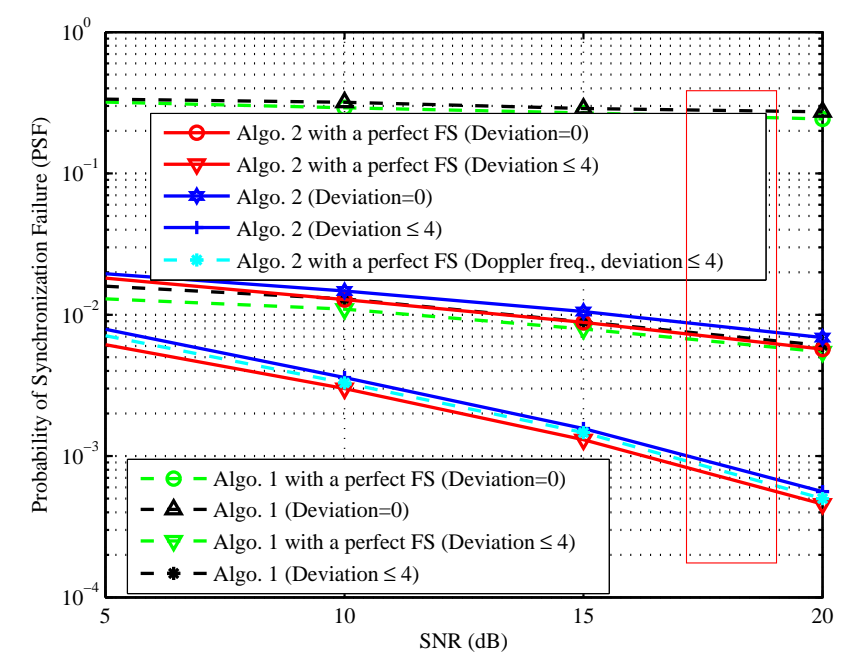

Figure 7. Probability of Synchronization Failure (the rectangular box presents the operating area of the 802.11a standard).

sated. When accepting arrival packets with deviation less than 4 samples, the PSF of both Algorithms 1 and 2 is reduced. However, the PSF of Algorithm 2 is smaller than that of Algorithm 1. Indeed at SNR $=17.5 \mathrm{~dB}$, when the $\mathrm{CFO}$ is not perfectly compensated we obtain PSF(Algo.2) $=1 \times 10^{-3}$ while PSF(Algo.1) $=7 \times 10^{-3}$.

We noted that the PSF performance of Algorithm 2 in both cases (i.e., perfect or not perfect FS) is almost the same. This is explained via Equation (31). If we replace $\mathbf{r}_{\Delta \theta_{m}}=\boldsymbol{\Phi}_{\Delta \theta_{m}, \epsilon} \mathbf{S}_{\Delta \theta_{m}} \mathbf{h}+\mathbf{g}$ into (31), we obtain

$$
\begin{aligned}
\widehat{\mathbf{h}}_{\Delta \theta_{m}}= & {\left[\mathbf{S}_{\Delta \theta_{m}}^{H} \mathbf{S}_{\Delta \theta_{m}}+\sigma_{g}^{2} \mathbf{R}_{\mathbf{h}}^{-\mathbf{1}}\right]^{-1} \mathbf{S}_{\Delta \theta_{m}}^{H} \mathbf{I}_{\Delta \theta, \hat{\epsilon}_{m}}\left(\mathbf{S}_{\Delta \theta_{m}} \mathbf{h}\right)+} \\
& {\left[\mathbf{S}_{\Delta \theta_{m}}^{H} \mathbf{S}_{\Delta \theta_{m}}+\sigma_{g}^{2} \mathbf{R}_{\mathbf{h}}^{-\mathbf{1}}\right]^{-1} \mathbf{S}_{\Delta \theta_{m}}^{H} \mathbf{\Phi}_{\Delta \theta_{m}, \hat{\epsilon}_{m}}(\mathbf{g}) }
\end{aligned}
$$

where $\mathbf{I}_{\Delta \theta, \hat{\epsilon}_{m}}$ is the diagonal matrix of size $\left(2 N+N_{G}+\right.$ $\left.N_{S}\right) \times\left(2 N+N_{G}+N_{S}\right)$ and is given by

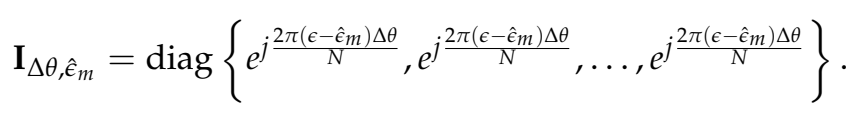


At SNR $=17.5 \mathrm{~dB}$, we have MSE(Algo. 2) $=1.6 \times 10^{-6}$ (see Figure 6). The experimental results show that the remaining value $\Delta \theta$ is relatively small and thus $e^{j 2 \pi\left(\epsilon-\hat{\epsilon}_{m}\right) \Delta \theta / N} \approx 1$. Therefore, $\mathbf{I}_{\Delta \theta, \hat{\epsilon}_{m}}$ is considered as an identity matrix. The first term in Equation (35) is therefore independent of the frequency offset. This explains why for Algorithm 2 the PSF when the CFO is not perfectly compensated is close to the one of the perfect FS.

One might ask how the performance of our algorithm is affected when the estimated channel $\widehat{\mathbf{h}}$ during the negotiation of the transmission medium (see Section 4.2.1, Equation (18)) has been slightly modified according to the walking speed of the receiving station just when the DATA is transmitted. In fact, if the CtS control frame has been correctly received, the interval time $\Delta t$ (see Figure 4) calculated by the difference between the starting time of the transmitted DATA and the RtS control frame is equal to $124 \mu$ s (i.e., $T_{\mathrm{RtS}}+T_{\mathrm{CtS}}+2 T_{\mathrm{SIFS}}$ ) when the rate is set to $6 \mathrm{Mb} / \mathrm{s}$ (the worst case) [16]. If we assume that the walking speed is equal to $1.5 \mathrm{~m} / \mathrm{s}$ with a carrier frequency of $5.2 \mathrm{GHz}$, the maximum Doppler frequency $f_{D}$ is a equal to $26 \mathrm{~Hz}$ which is a small value. Indeed each $j$-th channel tap is multiplied by $e^{j 2 \pi f_{D} \Delta t} \approx e^{j 0.02}$ and this does not effect the estimated channel $\widehat{h}$. This is confirmed by the simulation results provided by Figure 7 .

\section{Conclusion}

This paper proposed a novel algorithm for time and frequency synchronization conform to the IEEE 802.11a wireless communication standard. Investigations were focused first on finding information that could be exploited by the receiver at the physical frame. The SIGNAL field is retained since its parts are predictable when the CSMA/CA mechanism is activated and control frames RtS/CtS are exchanged during the negotiation of the transmission medium between stations before transmitting the DATA. In addition to the usual training sequences, the SIGNAL field is then used for improving the synchronization process. A new joint time and frequency synchronization strategy has been developed. The results have showed that the proposed synchronization algorithm improves the synchronization performance as compared to existing algorithms.
[5] J. J. van de Beek, M. Sandell, M. Isaksson, and P. O. Borjesson, "Lowcomplex frame synchronization in ofdm systems," in Fourth IEEE International Conference on Universal Personal Communications, Nov 1995, pp. 982-986.

[6] T. M. Schmidl and D. C. Cox, "Robust frequency and timing synchronization for OFDM," IEEE Transactions on Communications, vol. 45, no. 12, pp. 1613-1621, Dec 1997.

[7] B. Y. Prasetyo, F. Said, and A. H. Aghvami, "Fast burst synchronisation technique for OFDM-WLAN systems," in IEE Proceedings-Communications, Oct 2000, pp. 292298.

[8] H. Nguyen-Le and T. Le-Ngoc, "Pilot-aided joint cfo and doubly-selective channel estimation for OFDM transmissions," IEEE Transactions on Broadcasting, vol. 56, no. 4, pp. 514-522, Dec 2010.

[9] S. K. Manusani, R. S. Kshetrimayum, and R. Bhattacharjee, "Robust time and frequency synchronization in OFDM based 802.11a WLAN systems," in Annual IEEE Conference, India, Sept 2006, pp. 1-4.

[10] M. J. Canet, V. Almenar, J. Marin-Roig, and J. Valls, "Time synchronization for the IEEE 802.11a/g WLAN standard," in IEEE 18th International Symposium on Personal, Indoor and Mobile Radio Communications, (PIMRC), 2007, pp. 1-5.

[11] Y. Zhang, J. Zhang, and M. Xia, "Joint timing synchronization and channel estimation for OFDM systems via MMSE criterion," in IEEE 68th Vehicular Technology Conference, (VTC 2008-Fall), 2008, pp. 1-4.

[12] C. L. Nguyen, A. Mokraoui, P. Duhamel, and N. LinhTrung, "Time synchronization algorithm in IEEE 802.11a communication system," in Proceedings of the 20th European Signal Processing Conference (EUSIPCO), Aug 2012.

[13] — " "Enhanced time synchronization for IEEE 802.11a system using SIGNAL field and MAP channel estimation," in International Conference on Advanced Technologies for Communications (ATC), Oct 2012.

[14] P. J. Tourtier, R. Monnier, and P. Lopez, "Multicarrier modem for digital HDTV terrestrial broadcasting," Signal Processing: Image Communication, vol. 5, pp. 379-403, 1993.

[15] M. Speth, F. Classen, and H. Meyr, "Frame synchronization of OFDM systems in frequency selective fading channels," in IEEE 47th Vehicular Technology Conference, vol. 3, May 1997, pp. 1807-1811.

[16] M. Ergen and P. Varaiya, "Throughput analysis and admission control for IEEE 802.11a," Mobile Networks and Applications, vol. 10, pp. 705-716, 2005.

[17] Z. Li, A. Das, A. K. Gupta, and S. Nandi, "Full auto rate mac protocol for wireless ad hoc networks," in IEE Proceedings-Communications, vol. 152, 2005, pp. 311-319.

[18] O. Media, 802.11 Wireless Networks: The Definitive Guide, 2nd ed., 2005.

[19] F. R. Gfeller and W. Hirt, "Method for improved wireless optical communication and frames for use in a wireless optical communication system," U.S. Patent No 6,643,469, Nov 2003.

\section{REFERENCES}

[1] IEEE Std.802.11a, Std., 1999.

[2] T. Pollet, M. V. Bladel, and M. Moeneclaey, "BER sensitivity of OFDM systems to carrier frequency offset and Wiener phase noise," IEEE Transactions on Communications, vol. 43, no. 234, pp. 191-193, 1995.

[3] S. Ma, X. Pan, G.-H. Yang, and T.-S. Ng, “Blind symbol synchronization based on cyclic prefix for OFDM systems," IEEE Transactions on Vehicular Technology, vol. 58, no. 4, pp. 1746-1751, 2009.

[4] B. Park, E. Ko, H. Cheon, C. Kang, and D. Hong, "A blind ofdm synchronization algorithm based on cyclic correlation," in Proc. IEEE Global Telecommunication Conference, Nov 2001, pp. 3116-3119.

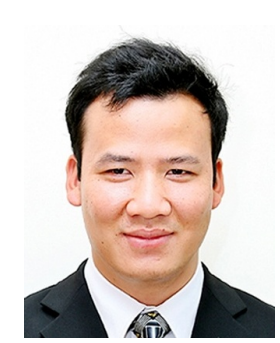

Cong Luong Nguyen received the B. E. and M. E. degree in Electronic and Telecommunication Engineering from Hanoi University of Science and Technology (HUST), in 2005 and 2007, respectively. Since 2011, he has been with Institut Galilée, Université Paris 13, Sorbonne Paris Cité as a Ph.D student. His research interests include wireless communication systems, SISO, MIMO systems, OFDM synchronization techniques, and related digital signal processing techniques 


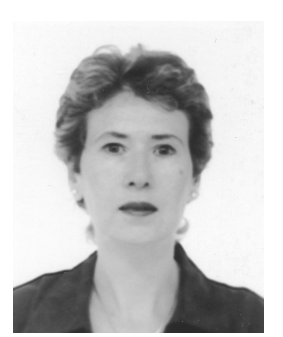

Anissa Mokraoui received the state engineering degree in electrical engineering from national telecommunication school in 1989 from Algeria, the M.S degree in information technology in 1990 and the Ph.D. degree in 1994 both from University Paris 11, Orsay France. From 92 to 94 , she worked at the National Institute of Telecommunications (INT, at Evry France) where her research activities were on digital signal processing, fast filtering algorithms and implementation problems on DSP. Since 1997, she is associate professor at Galilée Institute of Université Paris 13 Sorbonne Paris Cité, France. Her current research interests include image and video compression, image reconstruction, irregular sampling, interpolation, wavelet transforms, joint source-channelprotocol decoding and robust mobile transmission. She is co-author of more than 60 contributions to journals and conference proceedings. She served on program committees for conferences. She acts as a reviewer for many IEEE and EURASIP conferences and journals.

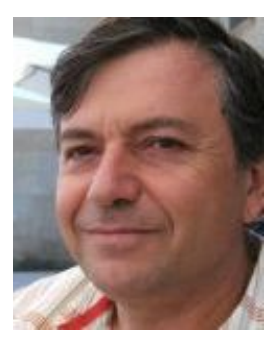

Pierre Duhamel was born in France in 1953. $\mathrm{He}$ received the Eng. Degree in Electrical Engineering from the National Institute for Applied Sciences (INSA) Rennes, France in 1975, the Dr. Eng. Degree in 1978, and the Doctoratès sciences degree in 1986, both from Orsay University, Orsay, France.

From 1975 to 1980, he was with ThomsonCSF (now Thales), Paris, France, where his research interests were in circuit theory and signal processing, including digital filtering and analog fault diagnosis. In 1980, he joined the National Research Center in Telecommunications (CNET, now Orange Labs), Issy les Moulineaux, France, where his research activities were first concerned with the design of recursive CCD filters. Later, he worked on fast algorithms for computing Fourier transforms and convolutions, and applied similar techniques to adaptive filtering, spectral analysis and wavelet transforms. From 1993 to Sept. 2000, he has been professor at ENST, Paris (National School of Engineering in Telecommunications) with research activities focused on Signal processing for Communications. He was head of the Signal and Image processing Department from 1997 to 2000. He is now with CNRS/L2S (Laboratoire de Signaux et Systemes, Gif sur Yvette, France), where he is developing studies in Signal processing for communications (including equalization, iterative decoding, multicarrier systems, cooperation) and signal/image processing for multimedia applications, including source coding, joint source-protocol-channel coding, and watermarking. He is currently investigating the connections between communication theory and networking.

Dr. Duhamel was chairman of the DSP committee from 1996 to 1998, a member of the SP for Com committee until 2001, and a member of the IEEE Signal Processing for Communications and Networking Technical Committee from 2004 to 2010. He was an associate Editor of the IEEE Transactions on Signal Processing from 1989 to 1991, an associate Editor for the IEEE Signal Processing Letters, and a guest editor for the special issue of the IEEE Trans. on SP on wavelets.

He was Distiguished lecturer, IEEE, for 1999, and was co-general chair of the 2001 International Workshop on Multimedia Signal Processing, Cannes, France. He was also co-technical chair of ICASSP 06, Toulouse, France, and of WCNC 2012, Paris, France. The paper on subspace-based methods for blind equalization, which he coauthored, received the "Best paper award" from the IEEE transactions on SP in 1998. He was awarded the "grand prix France Telecom" by the French Science Academy in 2000. He is Fellow of the IEEE since 1998 and of the EURASIP since 2008.

Dr Duhamel published more than 90 papers in international journals, more than 300 papers in international conferences, and holds 30 patents. He is a co-author of the book "Joint Source and Channel Decoding: A cross layer perspective with applications in video broadcasting" which appeared in 2009, Academic Press.

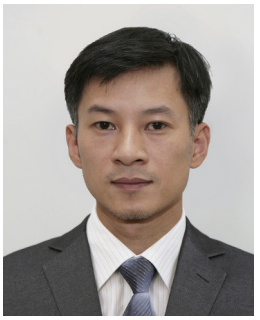

Nguyen Linh-Trung received both the B.Eng. and Ph.D. degrees in Electrical Engineering from Queensland University of Technology, Brisbane, Australia. From 2003 to 2005, he had been a postdoctoral research fellow at the French National Space Agency (CNES). In 2006, he joined the University of Engineering and Technology within Vietnam National University, Hanoi, and is currently an associate professor at its Faculty of Electronics and Telecommunications. He has held visiting positions at Telecom ParisTech, Vanderbilt University, Ecole Supérieure d'Electricité (Supelec) and the Université Paris 13 Sorbonne Paris Cité. His research focuses on methods and algorithms for data dimensionality reduction, with applications to biomedical engineering and wireless communications. The methods of interest include timefrequency analysis, blind source separation, compressed sensing, and network coding. He has published more than 40 scientific papers and a textbook on digital signal processing. He was co-chair of the technical program committee of the annual International Conference on Advanced Technologies for Communications (ATC) in 2011 and 2012. He is a senior member of the IEEE. 